\title{
Outcome of eribulin as a late treatment line for Thai metastatic breast cancer patients
}

This article was published in the following Dove Press journal: OncoTargets and Therapy

\author{
Chagkrit Ditsatham' \\ Imjai Chitapanarux ${ }^{2-4}$ \\ Areewan Somwangprasert ${ }^{1}$ \\ Kirati Watcharachan' \\ Panchaporn \\ Wongmaneerung' \\ Chaiyut Charoentum ${ }^{5}$ \\ Busyamas Chewaskulyong ${ }^{5}$ \\ Somvilai Chakrabandhu ${ }^{2}$ \\ Wimrak Onchan ${ }^{2}$

\section{Anongnart} \\ Teeyasuntranonn ${ }^{6}$ \\ Patumrat Sripan ${ }^{3}$ \\ 'Division of Head Neck Breast \\ Surgery, ${ }^{2}$ Division of Radiation \\ Oncology, ${ }^{3}$ Northern Thai Research \\ Group of Radiation Oncology, ${ }^{4}$ Chiang \\ Mai Cancer Registry, Maharaj Nakorn \\ Chiang Mai Hospital, ${ }^{5}$ Division of \\ Oncology, ${ }^{6}$ Pharmacy Division, \\ Maharaj Nakorn Chiang Mai Hospital, \\ Faculty of Medicine, Chiang Mai \\ University, Chiang Mai, Thailand
}

Correspondence: Imjai Chitapanarux Division of Radiation Oncology, Faculty of Medicine, Chiang Mai University, I 10 Intawarorose Road, Chiang Mai 50200, Thailand

Tel +6653935456

Fax +66 53935491

Email imjai.chitapanarux@cmu.ac.th
Background: We report the safety and efficacy of eribulin as a late treatment line in Thai metastatic breast cancer (MBC) patients.

Patients and methods: A total of $30 \mathrm{MBC}$ patients treated with eribulin between January 2014 and January 2017 were retrospectively analyzed. The patients were scheduled to receive $1.4 \mathrm{mg} / \mathrm{m}^{2}$ of eribulin on day 1 , day 8 and subsequently every 21 days. All patients had previously received at least three chemotherapy regimens including anthracycline and taxane. Response rate and progression-free survival (PFS) were analyzed.

Results: The median age was 56 years (range, 40-74 years), with a median follow-up time of 5.7 months (range, 0.2-25 months). The overall response rate was 30\% (nine patients): four patients had triple-negative breast cancer, three patients had luminal B breast cancer and two patients had luminal A breast cancer. The median PFS was 2.9 months (range, 0.2-14 months). The median number of previous chemotherapy regimens was 4 (range, 3-9). Univariate analysis showed that the number of regimens (four or fewer) prior to eribulin was statistically associated with superior PFS $(P=0.009)$. Multivariate analysis also showed similar statistical association between number of prior regimens (four or fewer) and better PFS adjusted by age group ( $\geq 50$ years; hazard ratio $=1.29 ; 95 \% \mathrm{CI}: 1.0-1.65 ; P=0.046$ ). There were no toxic deaths or grade 4 toxicities. Nine (30\%) patients had grade 3 anemia toxicities, and the other common toxicities were leukopenia and neutropenia. Four (13\%) patients required dose reduction and $16(53 \%)$ patients required dose delay because of toxicities.

Conclusion: Eribulin is an effective drug for heavily pretreated MBC patients with tolerable toxicities. The benefit was superior in patients who received fewer than four previous chemotherapy regimens.

Keywords: eribulin, metastatic breast cancer, late treatment line

\section{Introduction}

Metastatic breast cancer (MBC) is an incurable disease with a limited survival. Only $20 \%-30 \%$ of patients had long term 5-year survival. ${ }^{1}$ Treatment goals of this disease are to prolong survival and improve quality of life. ${ }^{2}$ Previous studies of capecitabine, gemcitabine and paclitaxel have demonstrated single-agent chemotherapy response rates. ${ }^{3-5}$ Eribulin, one of the modern chemotherapeutic agents, is a nontaxane synthetic microtubule dynamic inhibitor and an analog of the halichondrin B derived from marine sponge. Microtubules have an important role in the cell division process, the disturbance of which result in nonreversible mitotic blockage, which causes cellular death. In vitro and in vivo studies involving eribulin have shown a change in transitional morphology between mesenchymal and epithelial phenotypes, epithelial-mesenchymal transition (EMT) and mesenchymal-epithelial transition of breast cancer cells. ${ }^{25-27}$ The effect of these changes has resulted in a decrease in migration and invasiveness. Furthermore, 
eribulin results in a change in abnormal vascularization of the tumor to functional vessels, leading to an improvement in tumor perfusion; therefore, perfusion of the drug into the tumor cells is greater, decreasing the aggressiveness of the cells and reducing metastasis of breast cancer cells..$^{25-27}$ Eribulin showed an improvement in overall survival of pretreated MBC patients in the Phase III eribulin monotherapy versus treatment of physician's choice in patients with metastatic breast cancer (EMBRACE) trial, ${ }^{6,7}$ and it was approved for use as monotherapy in MBC patients previously given at least two prior chemotherapeutic regimens including anthracycline and taxane. ${ }^{8}$ To preserve the quality of life and tolerability of patients without compromising efficacy of the treatments, sequential monotherapies are preferable to combination regimens in most clinical MBC scenarios. ${ }^{8}$ In Thailand, eribulin has been approved for use in MBC patients since 2014. We conducted this retrospective study in pretreated MBC patients to evaluate the clinical benefit and tolerance of the drug in the real-world practice in Thai patients.

\section{Patients and methods}

This study is a retrospective review of MBC patients treated with eribulin between January 2014 and January 2017. This study was approved by the ethics committee of Faculty of Medicine, Chiang Mai University, and the committee waived the requirement for consent from patients, as it is a retrospective study. Only de-identified patient data were used in our study. The patients were scheduled to receive $1.4 \mathrm{mg} / \mathrm{m}^{2}$ of eribulin intravenously for 2-5 minutes as a monotherapy on day 1 , day 8 and every 21 days with schedule adjustment or dose modification due to intolerable toxicity of the treatment as necessary. All patients had previously received at least three chemotherapy regimens for the advanced disease including at least one of anthracycline or taxane. Patients who received eribulin for at least one cycle were included for analysis. Tumor response rates were measured using response evaluation criteria in solid tumors (RECIST) criteria ${ }^{9}$ or by clinical examination. Toxicity was recorded as per National Cancer Institute (NCI) Common Terminology Criteria for Adverse Events (CTCAE), version 4.0. ${ }^{10}$ Patients who received at least one dose of eribulin were included for toxicity analysis. Statistical analysis was done using commercial statistical software Stata 11.0 (StataCorp LP, College Station, TX, USA). The results of this study are reported in percentages for descriptive categorical data and as medians with corresponding interquartile ranges for continuous variables. $P$-values $<0.05$ were considered as statistically significant. Progression-free survival (PFS) was calculated using the Kaplan-Meier method, and the log-rank test was performed for significance test of the predicted factors that affect the prognosis.

\section{Results \\ Demographics and baseline clinical characteristics}

As shown in Table 1, $30 \mathrm{MBC}$ patients having received eribulin were included in the study. Their median age was 56 years (range, 40-74 years). All patients were divided into age range (years) as $36-40=1,41-45=0,46-50=5$, $51-55=8,56-60=10,61-65=3,66-70=2$ and $71-75=1$. Among them, five (17\%) were histological type of luminal A, eight $(27 \%)$ were of luminal B, six $(20 \%)$ were of HER2 positive and $11(36 \%)$ were of triple negative (TN). Median follow-up time was 5.7 months (range, 0.2-25 months). The median number of previous chemotherapy regimens was 4 (range, 3-9).

\section{Efficacy}

Overall clinical efficacy outcomes following eribulin treatment are shown in Table 2. The eribulin efficacy by age range (years) was 36-40=1 dead of disease (DD); $41-45=0 ; 46-50=4$ living with disease (LD), 1 DD; 51-55 = 4 LD, 4 DD; 56-60 = 6 LD, 4 DD; 61-65 =2 LD, $1 \mathrm{DD} ; 66-70=1 \mathrm{LD}, 1 \mathrm{DD}$ and $71-75=1 \mathrm{DD}$. The clinical benefit rate (response and stable disease) was 53\% (16/30) with partial response seen in nine $(30 \%)$ patients and stable disease seen in seven (23\%) patients. Progressive disease was reported in 14 (47\%) patients. Median PFS was 2.9 months (95\% CI: 2.0-4.4 months; Figure 1). Both our univariate and multivariate analyses showed a statistical association between the number of prior regimens and the PFS. Figure 2 shows that patients with four or fewer previous regimens to have a longer PFS, ie, 4.2 months (median PFS,

Table I Patients and cancer characteristics

\begin{tabular}{ll}
\hline Covariates & $\begin{array}{l}\text { Overall }(\mathbf{N}=\mathbf{3 0}) \\
\mathbf{n}(\%)\end{array}$ \\
\hline Age (years) & \\
$\quad$ Median age (IQR) & $56(52-59)$ \\
$<50$ & $4(13)$ \\
$\geq 50$ & $26(87)$ \\
Subtypes & \\
$\quad$ Luminal A & $5(17)$ \\
Luminal B & $8(27)$ \\
HER2 & $6(20)$ \\
$\quad$ TN & $11(36)$ \\
Number of chemotherapies prior to eribulin & \\
$\leq 4$ & $15(50)$ \\
$>4$ & $15(50)$ \\
Number of metastatic sites & \\
$<3$ & $14(47)$ \\
$\geq 3$ & $16(53)$ \\
\hline
\end{tabular}

Abbreviations: IQR, interquartile range; TN, triple negative. 
Table 2 Univariable and multivariable Cox proportional hazard regression analyses

\begin{tabular}{|c|c|c|c|c|c|c|}
\hline \multirow[t]{2}{*}{ Covariates } & \multicolumn{3}{|c|}{ Univariable analysis } & \multicolumn{3}{|c|}{ Multivariable analysis } \\
\hline & HR & $95 \% \mathrm{Cl}$ & $P$-value & aHR & $95 \% \mathrm{Cl}$ & $P$-value \\
\hline Age (years) & & & 0.100 & & & 0.134 \\
\hline$<50$ & 1.00 & & & 1.00 & & \\
\hline$\geq 50$ & 2.49 & $0.74-8.40$ & & 2.54 & $0.75-8.62$ & \\
\hline Tumor subtype & & & 0.040 & & & \\
\hline Luminal A & 1.00 & & & & & \\
\hline Luminal B & 0.35 & $0.11-1.16$ & & & & \\
\hline HER2 & 2.14 & $0.61-7.53$ & & & & \\
\hline $\mathrm{TN}$ & 0.65 & $0.22-1.92$ & & & & \\
\hline $\mathrm{TN}$ & & & 0.500 & & & \\
\hline No & 1.00 & & & & & \\
\hline Yes & 1.33 & $0.58-3.02$ & & & & \\
\hline Number of chemotherapies & & & 0.009 & & & 0.046 \\
\hline prior to eribulin & & & & & & \\
\hline$\leq 4$ & 1.00 & & & 1.00 & & \\
\hline$>4$ & 2.87 & $1.30-6.33$ & & 1.29 & $1.00-1.65$ & \\
\hline Number of metastatic sites & & & 0.630 & & & \\
\hline$<3$ & 1.00 & & & & & \\
\hline$\geq 3$ & 0.83 & $0.40-1.75$ & & & & \\
\hline
\end{tabular}

Abbreviations: $\mathrm{HR}$, hazard ratio; aHR, adjusted hazard ratio; TN, triple negative.

95\% CI: $2.5-6.0, P$-value $=0.009)$ vs 2.0 months $($ median PFS, 95\% CI: 0.9-3.0). Adjusted by age group ( $>50$ years), the multivariate analysis also showed patients with four or fewer regimens prior to eribulin to have better PFS (hazard ratio $=1.29 ; 95 \% \mathrm{CI}: 1.0-1.65, P=0.046)$. There was no statistical difference of eribulin efficacy between TN and non-TN subtypes.

\section{Adverse events (AEs)}

We used NCI CTCAE, version 4.0 for grading of toxicities. ${ }^{10}$ As shown in Table 3, most of the patients (20/30) had experienced grade 1 leukopenia (white blood cell [WBC] $\left.<3,000 / \mathrm{mm}^{3}\right)$. In all, nine $(30 \%)$ and three $(10 \%)$ patients had grade 3 anemia and leukopenia, respectively

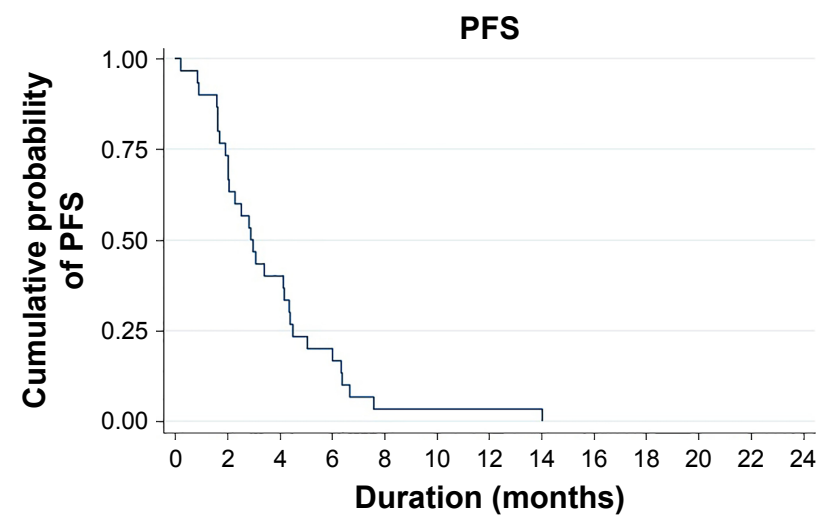

Figure I Kaplan-Meier estimates of PFS.

Abbreviation: PFS, progression-free survival. (grade 3 anemia: hemoglobin 6.5-8.0 g/dL and grade 3 leukopenia: $\left.1,000-2,000 / \mathrm{mm}^{3}\right)$. None of the patients had grade 4 toxicity or fatal AE (grade 4 anemia: hemoglobin $<6.5 \mathrm{~g} / \mathrm{dL}$, grade 3 leukopenia: hemoglobin $<1,000 / \mathrm{mm}^{3}$ ). There was no statistical association between hematologic toxicities and the numbers of chemotherapies prior to eribulin. Four (13\%) patients required dose-reduction from 1.4 to $1.1 \mathrm{mg} / \mathrm{m}^{2}$ and $16(53 \%)$ patients required dose delay because of bone marrow toxicities (Table 3). Nonhematologic toxicities were less common in this cohort of patients, and only grade 1 nausea, vomiting and malaise were reported in five $(16 \%)$ patients. All patients had alopecia from previous

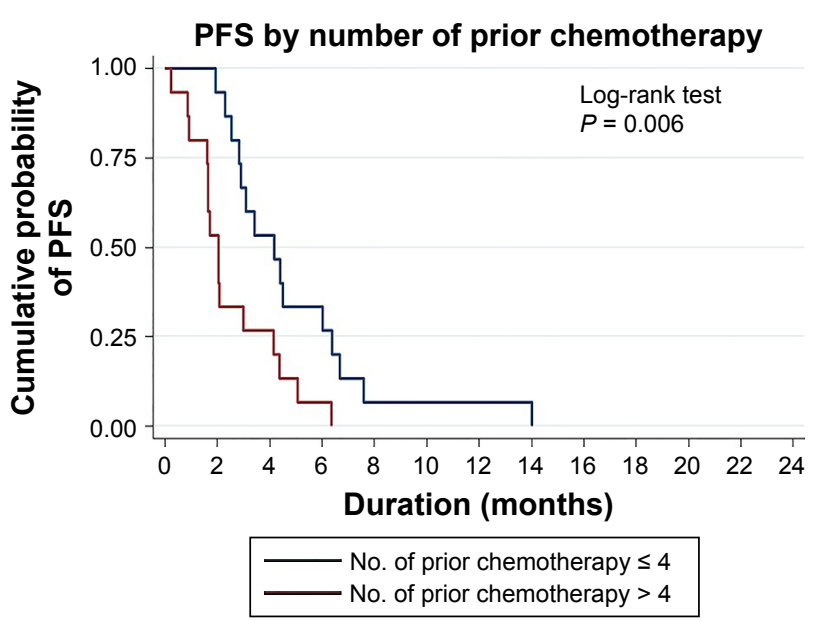

Figure 2 Kaplan-Meier estimates of PFS subgroup analysis between different numbers of prior chemotherapies.

Abbreviation: PFS, progression-free survival. 
Table 3 Hematologic AEs

\begin{tabular}{|c|c|c|c|c|}
\hline \multirow[t]{2}{*}{ Covariates } & \multicolumn{2}{|c|}{$\begin{array}{l}\text { Number of } \\
\text { chemotherapies } \\
\text { prior to eribulin }\end{array}$} & \multirow[t]{2}{*}{$\begin{array}{l}\text { Total } \\
(\mathbf{N}=\mathbf{3 0})\end{array}$} & \multirow[t]{2}{*}{$P$-value } \\
\hline & $\leq 4$ & $>4$ & & \\
\hline Leukopenia, n (\%) & & & & $>0.999$ \\
\hline Grade I & $10(67)$ & $10(67)$ & $20(67)$ & \\
\hline Grade 2 & $3(20)$ & $4(27)$ & $7(23)$ & \\
\hline Grade 3 & $2(13)$ & I (6) & $3(10)$ & \\
\hline Anemia, n (\%) & & & & 0.330 \\
\hline Grade I & $6(40)$ & $2(13)$ & $8(27)$ & \\
\hline Grade 2 & $5(33)$ & $8(54)$ & $13(43)$ & \\
\hline Grade 3 & $4(27)$ & $5(33)$ & $9(30)$ & \\
\hline
\end{tabular}

Abbreviation: $\mathrm{AE}$, adverse event.

chemotherapy when they started eribulin. Patients who experienced myelosuppression, length of this event was between 1 and 21 days (median 14 days).

\section{Discussion}

Eribulin is approved for use in the treatment of MBC patients who have experienced at least two chemotherapeutic regimens previously, and they should have been pretreated with anthracycline or taxane because of the improvement on overall survival from EMBRACE study. ${ }^{6}$ Standard dosage of eribulin mesylate is $1.4 \mathrm{mg} / \mathrm{m}^{2}$ for day 1 , day 8 and every 21 days in each cycle, but for patient experiencing toxicities, the dose may be delayed or modified to 1.1 or $0.7 \mathrm{mg} / \mathrm{m}^{2}$ depending on severity. ${ }^{6}$ The most common AEs in our study were hematologic toxicities, which included leukopenia, neutropenia and anemia, which were similar to those of EMBRACE and other studies. ${ }^{6,11-13}$ However, there was no any grade 4 hematologic toxicities reported in this study. The dose modification was found in $13 \%$ of patients and delayed drug administration in $53 \%$ of patients due to hematologic toxicities, which were similar to those treated with eribulin in Asian patients as reported in Japan and India. ${ }^{11,14,15}$ Having shown an improvement in the overall survival in the EMBRACE study, ${ }^{6}$ eribulin is approved for use in the treatment of MBC patients previously treated with two or more chemotherapeutic regimens. These prior regimens should include anthracycline or taxane. Statistically, we did not find any difference in the response to eribulin between TN subtype breast cancer patients and non-TN breast cancer patients. A median PFS at 2.90 months (95\% CI: 2.0-4.4 months) in our study was comparable to that reported in several other studies that used monotherapy in the late line regimens, eg, 4.9 months for capecitabine, ${ }^{16} 3.7$ months for vinorelbine ${ }^{17}$ and 3.1 months for ixabepilone. ${ }^{18}$
When analyzing the impact of number of prior regimens before eribulin, we found that the patients who had received four or fewer regimens had better PFS than those with a higher number of prior regimens, ie, 4 vs 2 months. This finding was not inferior to other regimens. ${ }^{19-22}$ The EMBRACE study reported a median PFS of 3.7 months for a median number of prior regimens of 4 , including neoadjuvant and adjuvant regimens. However, if we focused on the group of patients who received no more than four regimens as in the EMBRACE study, the corresponding median PFS was 4 months. Our study included patients who received at least three prior regimens that might attribute to the poor overall median PFS, but nonetheless in line with 2.5-4 months in other previous reports for heavily pretreated patients (four or more prior lines of treatment). ${ }^{8,11,13,23}$ The better PFS found in this study associated with fewer prior chemotherapy regimens in this study, which was similar to that reported in previous studies. ${ }^{12,24}$ Interestingly, four patients of the overall response rate (nine patients) were TN breast cancer, but more patients are needed to evaluate the efficacy between non-TN and TN patients. The reasons behind the more effective outcome of the use of eribulin as an earlier than late treatment line of treatment may be attributed to the nonmitotic effect on tumor biology by reversal of EMT affecting the tumor vasculature remodeling, which will increase tumor oxygenation, leading to a decreased capacity for metastasis and invasion..$^{25-27}$

\section{Conclusion}

Eribulin is an effective drug in heavily pretreated Thai MBC with tolerable toxicities. The benefit was better in patients who received eribulin as an earlier treatment.

\section{Disclosure}

The authors report no conflicts of interest in this work.

\section{References}

1. Kubista E. [Breast cancer: figures and facts]. Wien Med Wochenschr. 2001;151(21-23):548-551. German.

2. El Saghir NS, Tfayli A, Hatoum HA, Nachef Z, Dinh P, Awada A. Treatment of metastatic breast cancer: state-of-the-art, subtypes and perspectives. Crit Rev Oncol Hematol. 2011;80(3):433-449.

3. Kinoshita J, Haga S, Shimizu T, et al. Monotherapy with paclitaxel as third-line chemotherapy against anthracycline-pretreated and docetaxel-refractory metastatic breast cancer. Breast Cancer. 2002;9(2): 166-169.

4. Rha SY, Moon YH, Jeung HC, et al. Gemcitabine monotherapy as salvage chemotherapy in heavily pretreated metastatic breast cancer. Breast Cancer Res Treat. 2005;90(3):215-221.

5. Burstein HJ, Storniolo AM, Franco S, et al. A phase II study of lapatinib monotherapy in chemotherapy-refractory HER2-positive and HER2negative advanced or metastatic breast cancer. Ann Oncol. 2008; 19(6):1068-1074. 
6. Cortes J, O'Shaughnessy J, Loesch D, et al. Eribulin monotherapy versus treatment of physician's choice in patients with metastatic breast cancer (EMBRACE): a phase 3 open-label randomised study. Lancet. 2011;377(9769):914-923.

7. Twelves C, Awada A, Cortes J, et al. Subgroup analyses from a phase 3, open-label, randomized study of eribulin mesylate versus capecitabine in pretreated patients with advanced or metastatic breast cancer. Breast Cancer (Auckl). 2016;10:77-84

8. Digklia A, Voutsadakis IA. Eribulin for heavily pre-treated metastatic breast cancer patients. World J Exp Med. 2015;5(3):194-199.

9. Eisenhauer EA, Therasse P, Bogaerts J, et al. New response evaluation criteria in solid tumours: revised RECIST guideline (version 1.1). Eur $J$ Cancer. 2009;45(2):228-247.

10. Chen AP, Setser A, Anadkat MJ, et al. Grading dermatologic adverse events of cancer treatments: the Common Terminology Criteria for Adverse Events Version 4.0. J Am Acad Dermatol. 2012;67(5):1025-1039.

11. Bajpai J, Ramaswamy A, Gupta S, Ghosh J, Gulia S. Eribulin in heavily pretreated metastatic breast cancer: a tertiary care center experience from India. Indian J Cancer. 2016;53(3):460-463.

12. Fabi A, Moscetti L, Ciccarese M, et al. Eribulin in heavily pretreated metastatic breast cancer patients and clinical/biological feature correlations: impact on the practice. Future Oncol. 2015;11(3):431-438.

13. Poletti P, Ghilardi V, Livraghi L, Milesi L, Rota Caremoli E, Tondini C. Eribulin mesylate in heavily pretreated metastatic breast cancer patients: current practice in an Italian community hospital. Future Oncol. 2014; 10(2):233-239.

14. Ates O, Babacan T, Kertmen N, et al. Efficacy and safety of eribulin monotherapy in patients with heavily pretreated metastatic breast cancer. J BUON. 2016;21(2):375-381.

15. Aogi K, Iwata H, Masuda N, et al. A phase II study of eribulin in Japanese patients with heavily pretreated metastatic breast cancer. Ann Oncol. 2012;23(6):1441-1448.

16. Osako T, Ito Y, Takahashi S, Tokudome N, Iwase T, Hatake K. Intermittent capecitabine monotherapy with lower dose intensity in heavily pretreated patients with metastatic breast cancer. Tumori. 2007;93(2): 129-132.

17. Seo HY, Lee HJ, Woo OH, et al. Phase II study of vinorelbine monotherapy in anthracycline and taxane pre-treated metastatic breast cancer. Invest New Drugs. 2011;29(2):360-365.
18. Perez EA, Lerzo G, Pivot X, et al. Efficacy and safety of ixabepilone (BMS-247550) in a phase II study of patients with advanced breast cancer resistant to an anthracycline, a taxane, and capecitabine. $J$ Clin Oncol. 2007;25(23):3407-3414.

19. Yamamura J, Masuda N, Yamamoto D, et al. Gemcitabine and vinorelbine combination chemotherapy in taxane-pretreated patients with metastatic breast cancer: a phase II study of the Kinki Multidisciplinary Breast Oncology Group (KMBOG) 1015. Chemotherapy. 2017; 62(5):307-313.

20. Bajpai J, Ramaswamy A, Gupta S, Ghosh J, Gulia S. Everolimus in heavily pretreated metastatic breast cancer: is real world experience different? Indian J Cancer. 2016;53(3):464-467.

21. Uncu D, Bayoglu IV, Arslan UY, et al. Trastuzumab-based retreatment after lapatinib in heavily pretreated HER2 positive metastatic breast cancer: an anatolian society of medical oncology study. Asian Pac J Cancer Prev. 2015;16(9):4127-4131.

22. Hong JY, Park YH, Choi MK, et al. Characterization of durable responder for capecitabine monotherapy in patients with anthracyclineand taxane-pretreated metastatic breast cancer. Clin Breast Cancer. 2015;15(5):e287-e292.

23. Ramaswami R, O’Cathail SM, Brindley JH, Silcocks P, Mahmoud S, Palmieri C. Activity of eribulin mesylate in heavily pretreated breast cancer granted access via the Cancer Drugs Fund. Future Oncol. 2014; 10(3):363-376.

24. Lee DW, Teoh DC, Chong FL. Treatment of heavily pre-treated metastatic breast cancer with eribulin: first local experience in Sabah. Med J Malaysia. 2016;71(6):348-350.

25. Yoshida T, Ozawa Y, Kimura T, et al. Eribulin mesilate suppresses experimental metastasis of breast cancer cells by reversing phenotype from epithelial-mesenchymal transition (EMT) to mesenchymalepithelial transition (MET) states. Br J Cancer. 2014;110(6): 1497-1505.

26. Funahashi Y, Okamoto K, Adachi Y, et al. Eribulin mesylate reduces tumor microenvironment abnormality by vascular remodeling in preclinical human breast cancer models. Cancer Sci. 2014;105(10): 1334-1342.

27. Ueda S, Saeki T, Takeuchi H, et al. In vivo imaging of eribulin-induced reoxygenation in advanced breast cancer patients: a comparison to bevacizumab. Br J Cancer. 2016;114(11):1212-1218.
OncoTargets and Therapy

\section{Publish your work in this journal}

OncoTargets and Therapy is an international, peer-reviewed, open access journal focusing on the pathological basis of all cancers, potential targets for therapy and treatment protocols employed to improve the management of cancer patients. The journal also focuses on the impact of management programs and new therapeutic agents and protocols on

\section{Dovepress}

patient perspectives such as quality of life, adherence and satisfaction. The manuscript management system is completely online and includes a very quick and fair peer-review system, which is all easy to use. Visit http://www.dovepress.com/testimonials.php to read real quotes from published authors. 